\title{
Olfactory Function in Parkinson's Disease
}

\author{
Antje Haehner, ${ }^{1}$ Thomas Hummel ${ }^{2}$ and Heinz Reichmann ${ }^{3}$ \\ 1. Assistant Professor, Department of Otorhinolaryngology and Department of Neurology; 2. Professor, Department of Otorhinolaryngology; \\ 3. Professor of Neurology, Department of Neurology, University of Dresden Medical School
}

\begin{abstract}
Impairment of olfaction is a characteristic and early feature of Parkinson's disease (PD). Recent data indicate that $>95 \%$ of patients with PD present with significant olfactory loss. Deficits in sense of smell may precede clinical motor symptoms by years and can be used to assess risk of developing PD in otherwise asymptomatic individuals. This article summarises the available literature on olfactory function in PD, which indicates that olfactory tests improve the diagnostic process in these patients; however, it is still unclear which lesion causes olfactory loss in PD.
\end{abstract}

\section{Keywords}

Parkinson's disease, parkinsonism, smell, olfaction, diagnosis

Disclosure: The authors have no conflicts of interest to declare.

Received: 5 April 2010 Accepted: 25 May 2010 Citation: European Neurological Review, 2010;5(1):26-9 DOI:10.17925/ENR.2010.05.01.26

Correspondence: Antje Haehner, Department of Otorhinolaryngology, University of Dresden Medical School, Fetscherstr. 74, 01307 Dresden, Germany.

E: antje.haehner@uniklinikum-dresden.de

There is convincing evidence from numerous studies using both psychophysical and electrophysiological approaches that olfaction is markedly reduced in Parkinson's disease (PD). In light of the current data on smell loss in PD patients, olfactory dysfunction has to be seen as a cardinal symptom of the disease that is even more consistent than the classic symptom of tremor.' Consequently, structured and validated tests of olfactory function should be a mandatory part of the early and differential diagnosis of PD. Olfactory tests are quick and easy to use and testing can be performed in a reliable fashion even in non-specialised centres.

\section{Olfactory Loss in Parkinson's Disease}

The association between olfactory dysfunction and PD was noted more than 30 years ago. ${ }^{2}$ Virtually all studies performed since then have shown olfactory disturbances in PD patients. However, data on the prevalence of olfactory dysfunction in PD range from 45 and $49 \%$ in the pioneering studies of Ansari and Johnson ${ }^{2}$ and Ward, ${ }^{3}$ respectively, up to $74 \%$ in the work of Hawkes et al., ${ }^{4}$ or as high as $90 \%$ in a study published by Doty et al..$^{5}$ In our recent multicentre study ${ }^{6}$ using a comprehensive testing method in a large sample of PD patients $(n=400)$ from three independent populations, the prevalence of olfactory dysfunction in people with PD was greater than previously reported in terms of norms obtained in healthy young subjects. More than $96 \%$ of PD patients were found to present with olfactory dysfunction (see Figure 1). When using age-dependent normative criteria, $74.5 \%$ of this study population was diagnosed with olfactory loss (see Figure 2). Furthermore, $>80 \%$ of PD patients with smell loss were functionally anosmic or severely hyposmic regardless of the olfactory test being used for diagnosis. These data also confirmed numerous previous studies in terms of the missing correlation between olfactory loss and both duration of disease $e^{4,5,7}$ and the clinical severity of PD as measured by means of the Hoehn and Yahr scale and the Unified Parkinson's Disease Rating Scale (UPDRS), ${ }^{8}$ although some studies found a correlation between the severity of PD and certain measures of olfactory function, namely latencies of olfactory event-related potentials ${ }^{9}$ or results from an odour-discrimination task..$^{10} \mathrm{~A}$ follow-up study in 27 PD patients ${ }^{11}$ revealed that psychophysically tested olfactory function changes in an unpredictable manner. Thus, the olfactory deficit appears to be variable over time such that some patients develop anosmia while other patients convert from anosmia to hyposmia.

In terms of olfactory function, we did not find major differences between subtypes of PD, namely tremor-dominant PD, akinetic-rigid PD and mixed-type PD. While this confirms previous observations in a small sample of 37 patients, ${ }^{12}$ the current findings are in contrast to reports by Stern and colleagues, ${ }^{7}$ who reported significantly better odour identification scores in patients with tremor-predominant PD $(n=40)$ than in cases with postural instability gait disorderpredominant PD $(n=23)$. Although differences between studies may be due to the type of olfactory test used, sample size, normative data and age distribution (which varied between these investigations), available data allow the conclusion that olfactory dysfunction is a highly reliable symptom of the disease.

\section{Olfactory Dysfunction as an Early Symptom of Parkinson's Disease}

PD patients frequently report reduction in their sense of smell that occurs a few years prior to the onset of motor symptoms. However, the unawareness of patients of smell deficits may account for the inconsistent results described in retrospective surveys. In a small study ${ }^{13}$ on questioning prior to olfactory testing, nine of 37 patients (24\%) indicated an awareness of a decrease of olfactory function that 
actually preceded their diagnosis of PD. This is in a line with a number of other studies that could show evidence of olfactory dysfunction in untreated, newly diagnosed patients. ${ }^{5,14}$ Recent studies support the view that deficits in sense of smell may precede clinical motor symptoms by years. A study by Ponsen et al. ${ }^{15}$ on 361 asymptomatic relatives of $\mathrm{PD}$ patients selected 40 relatives with the lowest olfactory performance. Within two years of follow-up, $10 \%$ of these first-degree relatives of PD patients with significant olfactory loss developed clinical PD. In a follow-up study five years from baseline testing, ${ }_{1}{ }^{6}$ five relatives had developed clinical PD as defined by the United Kingdom Parkinson's Disease Society Brain Bank Diagnostic Criteria for Parkinson's Disease. Initial clinical (motor) symptoms appeared zero to 52 months (median 15 months) after baseline testing. Poorer performance on each of three olfactory processing tasks was associated with an increased risk of developing PD within five years. Among the olfactory tests used, odour-discrimination testing appeared to be the best predictor of the future development of PD.

In 2007, ${ }^{17}$ we published data on a clinical follow-up of a previous investigation ${ }^{18}$ in 30 patients diagnosed with idiopathic olfactory loss. Four years from baseline, $7 \%(n=2)$ of the individuals with idiopathic olfactory loss who were available for follow-up examination $(n=24)$ had newly developed clinical PD symptoms. Altogether, $13 \%(n=4)$ of the patients presented with PD-relevant abnormalities of the motor system. The results indicated that unexplained olfactory loss may be associated with an increased risk of developing PD relevant motor symptoms.

By contrast, authors of a twin study ${ }^{19}$ concluded that smell identification ability may not be a sensitive indicator of future PD, even in a theoretically at-risk population. This was based on the fact that patients who subsequently developed PD had no evidence of significant smell loss when they were initially tested. However, as pointed out by the authors themselves, the reason for this negative finding might lie in the very long delay of seven years between baseline assessment and follow-up visit. The initial test may have been too early for their subjects to have developed signs of smell dysfunction.

This is in accord with the results of a large longitudinal study by Ross and colleagues, ${ }^{20}$ who assessed olfactory function in 2,267 elderly men in the Honolulu Heart Program and found an association between smell loss and future development of PD. They came to the conclusion that impaired olfaction can pre-date PD by at least four years and may be a useful screening tool to detect those at high risk of development of PD in later life. This relationship appears to weaken beyond the four-year period. However, currently no accurate information is available on the initial onset of olfactory deterioration. Estimates for the duration of the prodromal phase range from two to 50 years depending on the methods of measurement. ${ }^{21}$

Recent data on olfactory loss as a PD symptom that is present in the earliest stages of the disease are compatible with predictions made on the basis of neuropathological investigations. Braak et al. ${ }^{22}$ describe involvement of olfactory pathways and lower brainstem before nigrostriatal pathways are affected, which might cause early non-motor symptoms. Huisman et al. ${ }^{23}$ found an increase of (inhibitory) dopaminergic neurons in the olfactory bulb in PD patients. They interpreted their finding within the context of a possible compensatory mechanism in response to the loss of dopaminergic

\section{Figure 1: Olfactory Function in Parkinson's Disease}

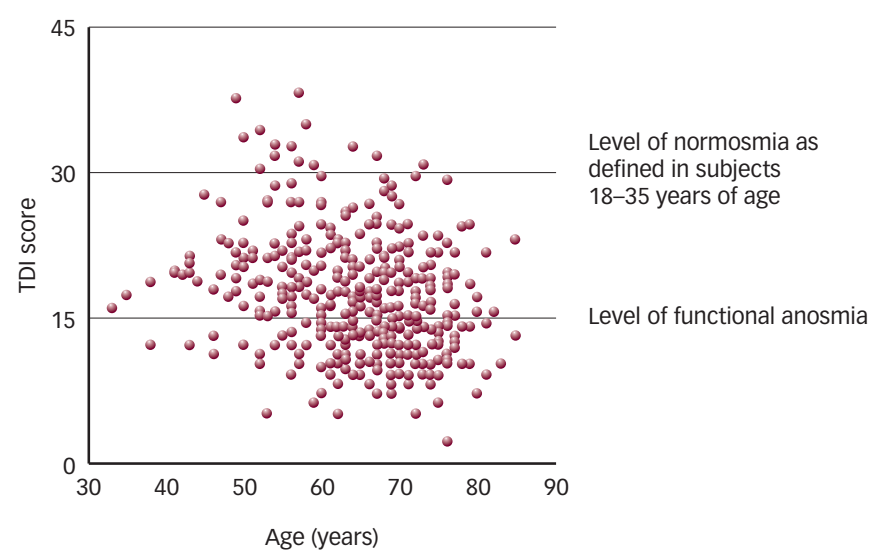

Olfactory function of a total of 400 Parkinson's disease (PD) patients. Results are shown as a composite TDI score (sum of odour threshold, odour discrimination and odour identification score) adjusted to age-independent criteria of hyposmia (olfactory function in a group of 18-35-year-old healthy subjects, considered to be the standard population in terms of normal olfactory sensitivity).

\section{Figure 2: Olfactory Function in Parkinson's Disease Adjusted to Age-related Norms}

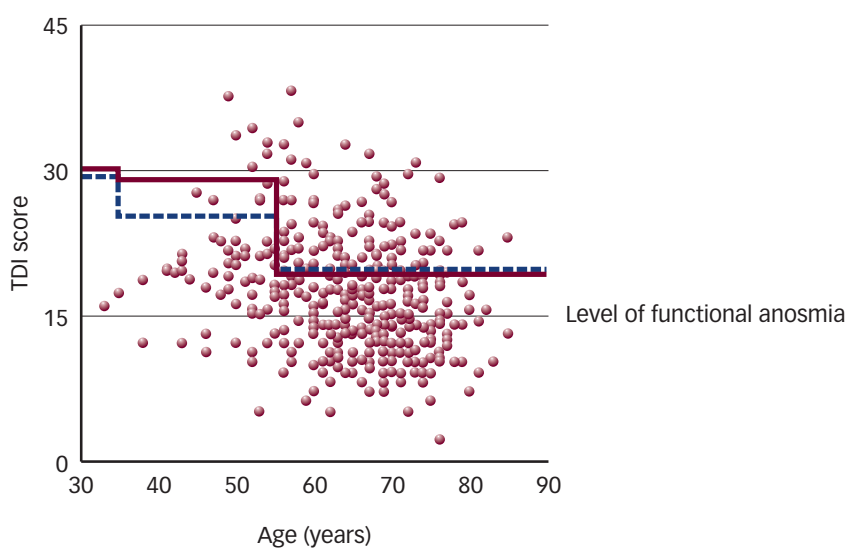

Age-related level of normosmia

Women aren

Olfactory function of a total of 400 Parkinson's disease (PD) patients. Results are shown as a composite TDI score (sum of odour threshold, odour discrimination and odour identification score) adjusted to age-related norms.

neurons in the basal ganglia. However, in 2008 they described gender-related differences in increase of dopaminergic neurones with a significantly lower number of tyrosine hydroxylase positive cells in control females compared with control males. ${ }^{24}$ As the number of dopaminergic cells in the olfactory bulbs of both female and male PD patients equals that of healthy males, the authors came to the conclusion that olfactory dysfunction in PD cannot simply be ascribed to dopamine in the olfactory bulb.

In summary, although the number of prospective studies in this field is still rather small, olfactory loss should be considered a promising contribution to the early diagnosis of PD.

\section{Olfactory Dysfunction in Differential Diagnosis}

Numerous studies suggest that olfactory disturbances in PD may have diagnostic utility for the differentiation of PD from other movement disorders. Wenning et al. ${ }^{25}$ presented data suggesting that olfactory function is differentially impaired in distinct parkinsonian syndromes (PS). They reported a preserved or mildly impaired olfactory function 
Figure 3: Olfactory Bulb Volumes in Parkinson's Disease

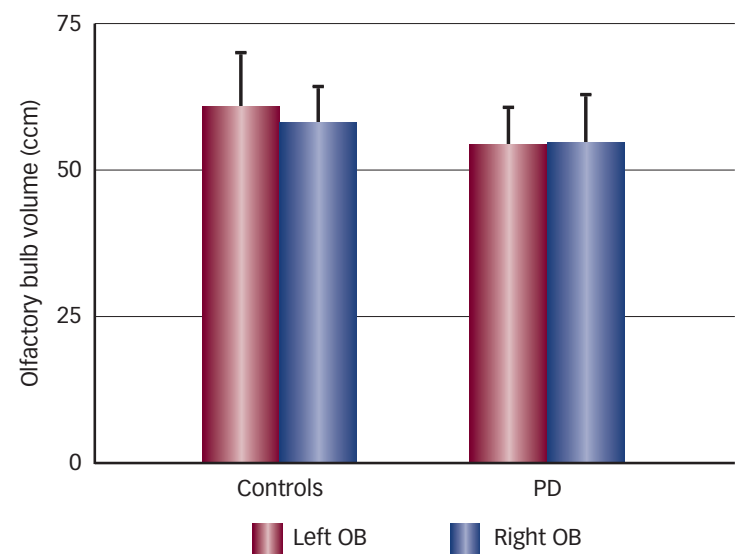

Olfactory bulb $(O B)$ volumes (standard error of the mean) separately for the left (red) and right side (blue) in patients with Parkinson's disease (PD) $(n=11)$ and healthy controls $(n=9)^{38}$

to be more likely for atypical parkinsonism such as multiple system atrophy (MSA; $n=29$ ), progressive supranuclear palsy (PSP; $n=15$ ) or corticobasal degeneration (CBD; $n=7$ ), whereas markedly pronounced olfactory loss appeared to suggest PD $(n=118)$. Similar to the results of Wenning et al., in a study on 50 parkinsonian patients ${ }^{12,13}$ we also found evidence for olfactory loss in MSA $(n=8)$, but little or no olfactory loss in (the few investigated) patients with PSP $(n=2)$ and CBD $(n=1)$ Nineteen PD patients were found to suffer from anosmia and another 13 had severe hyposmia, while five showed moderate hyposmia. In terms of the differentiation between MSA and PD at a cut-off of a TDI score (combined results for odour thresholds, odour discrimination and odour identification; see also reference 26) of 19.5, psychophysical testing had a sensitivity of $78 \%$ and a specificity of $100 \%$. When the cut-off TDI score was increased to 24.8 , sensitivity in this sample was $100 \%$ while specificity fell to $63 \%$. This moderate specificity seems to be the limiting parameter for diagnostic purposes. A recent American Academy of Neurology practice parameter on the diagnosis and prognosis of PD concluded that olfactory testing "should be considered" to differentiate PD from PSP and CBD but not from MSA. ${ }^{27}$ Furthermore, Liberini et al. ${ }^{28}$ reported a significant olfactory impairment in Lewy body disease (LBD), which does not allow differentiation from PD. In a sample of 116 patients with mild LBD, mild Alzheimer's disease, mild cognitive impairment and controls, Williams et al. ${ }^{29}$ describe even more marked olfactory impairment in patients with mild dementia with Lewy bodies than in those with mild Alzheimer's disease. This lends significance to the role of Lewy body pathology in olfactory dysfunction ${ }^{30}$ that would be in line with the observation that patients with non-degenerative causes of parkinsonism such as vascular parkinsonism ${ }^{31}$ present with preserved smell function. There is also evidence for less olfactory disturbance in familial parkinsonism. In PARK2 the olfactory sense is relatively well preserved whereas PARK1 subjects are mildly hyposmic. ${ }^{31}$ Recent data $^{32}$ suggested that PARK8 individuals present with impaired olfactory identification, while the small number of tested asymptomatic carriers showed normal olfactory performance.

1-methyl-4-phenyl-1,2,3,6 tetrahydropyridine (MPTP)-induced parkinsonism is generally thought not to affect olfaction. ${ }^{33}$ However, Miwa et al. ${ }^{34}$ demonstrated that MPTP causes both PD-like symptoms and olfactory disturbance in animals. We found an association between medication-induced parkinsonism and olfactory dysfunction in patients with psychotic depression treated with D2-blocking neuroleptic drugs. ${ }^{35}$ Here, the severity of motor symptoms was positively correlated with the degree of olfactory dysfunction, which might indicate patients with a latent basal ganglia dysfunction. Similar to the results seen in drug-induced parkinsonism, data from a recent study reveal that Wilson's disease patients with neurological symptoms show a significant olfactory dysfunction compared with hepatic-type patients. ${ }^{36}$ Individuals who are more severely neurologically affected also present with more pronounced olfactory deficits. Based on these observations, olfactory testing should not be considered to differentiate PD from these specific conditions. However, olfactory testing has been shown to be important in cases where patients present with parkinsonian features but preserved olfaction; here the diagnosis of PD should be re-considered.

\section{Structural and Functional Studies of the Olfactory System in Parkinson's Disease}

It is still unclear which lesion causes olfactory loss in PD. There is little information on structural changes at the level of the olfactory epithelium. Recently, we compared bioptic material from PD patients with that from patients with olfactory dysfunction due to other reasons, e.g. congenital anosmia or post-infectious olfactory loss. ${ }^{37}$ However, the results of the study did not reveal specific histological or histochemical changes in the nasal mucosa of PD patients. Therefore, PD-related olfactory impairment seems not to be directly associated with specific changes in the olfactory epithelium. In terms of volumetrics of the olfactory bulb (OB), results indicated that there is little or no difference between PD patients and healthy controls in terms of OB volume (see Figure 3). ${ }^{38}$ However, in a study by Scherfler et al., ${ }^{39}$ voxel-wise analysis of diffusion-weighted imaging revealed disruption of the olfactory tract in PD compared with age-matched healthy controls. Finally, studies based on functional magnetic resonance imaging (MRI) suggested that neuronal activity in the amygdala and hippocampus is reduced in PD patients compared with controls, which may specifically affect olfactory function. ${ }^{40}$ In addition, neuronal activity in components of cortico-striatal loops appears to be upregulated, indicating compensatory processes involving the dopaminergic system. Results from Wattendorf et al. ${ }^{4}$ indicated that cortical atrophy in olfactory regions correlate with olfactory dysfunction. Thus, it seems that cerebral changes are the basis of the olfactory loss observed in PD patients.

Currently, none of the structural measurements of the olfactory system mentioned above is routinely used. Consequently, validated psychophysical tests of olfactory function should be a mandatory part of the early and differential diagnosis of PD.

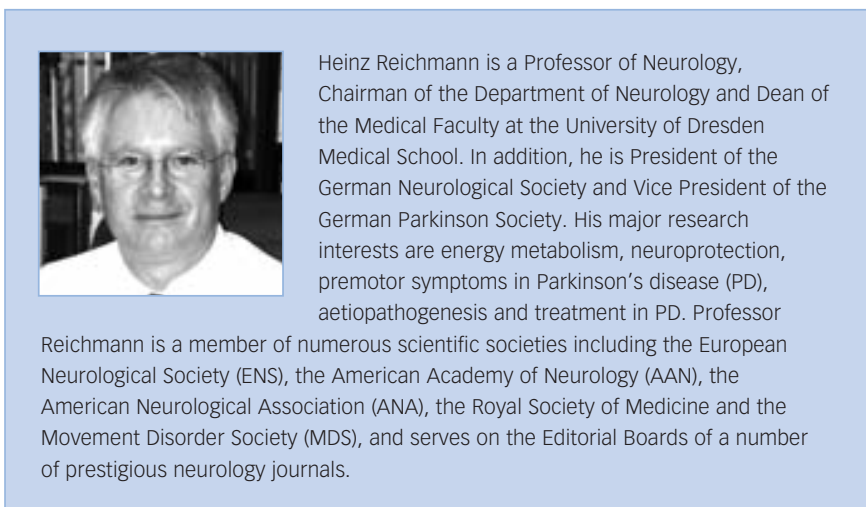


1. Hoehn MM, Yahr MD, Parkinsonism. Onset, progression and mortality, Neurology, 1967;17:427-42.

2. Ansari $K A$, Johnson $A$, Olfactory function in patients with Parkinson's disease, J Chron Dis, 1975;28:493-7.

3. Ward CD, Hess WA, Calne DB, Olfactory impairment in Parkinson's disease, Neurology, 1983;33:943-6.

4. Hawkes $\mathrm{CH}$, Shephard BC, Daniel SE, Olfactory dysfunction in Parkinson's disease, J Neurol Neurosurg Psychiatry, 1997;62:36-46.

5. Doty RL, Deems D, Steller S, Olfactory dysfunction in Parkinson's disease: A general deficit unrelated to neurologic signs, disease stage, or disease duration, Neurology, 1988;38:1237-44.

6. Haehner A, Boesveldt S, Berendse HW et al., Prevalence of smell loss in Parkinson's disease - a multicenter study, Parkinsonism Relat Disord, 2009;15:490-94.

7. Stern MB, Doty RL, Dotti M, et al., Olfactory function in Parkinson's disease subtypes, Neurology, 1994;44:266-8.

8. Ramaker C, Marinus J, Stiggelbout AM, et al., Systematic evaluation of rating scales for impairment and disability in Parkinson's disease, Mov Disord, 2002;17:867-76.

9. Hummel T, In: Chase T, Bedard P (Eds), Focus on Medicine Vol. 14 - New developments in the drug therapy of Parkinson's disease. Blackwell Science, Oxford, UK, 1999;47-53.

10. Tissingh G, Berendse HW, Bergmans P, et al., Loss of olfaction in de novo and treated Parkinson's disease: possible implications for early diagnosis, Mov Disord, 2001;16:41-6.

11. Herting B, Scholz S, Haehner A, et al., A longitudinal study of olfactory function in patients with idiopathic Parkinson's disease, J Neurol, 2008;255:367-70.

12. Müller $A$, Müngersdorf $M$, Reichmann $H$, et al., Olfactory function in Parkinsonian syndromes, J Clin Neurosci, 2002;9:521-4.

13. Müller $A$, Reichmann $H$, Livermore $A$, et al., Olfactory function in idiopathic Parkinson's disease (IPD): results from cross-sectional studies in IPD patients and long-term followup of de-novo IPD patients, J Neural Transm, 2002;109:805-11.

14. Tissingh G, Berendse HW, Bergmans P, et al., Loss of olfaction in de novo and treated Parkinson's disease: possible implications for early diagnosis, Mov Disord, 2001;16:41-6.

15. Ponsen MM, Stoffers D, Booij J, et al., Idiopathic hyposmia as a preclinical sign of Parkinson's disease, Ann Neurol, 2004;56:173-81.
16. Ponsen MM, Stoffers D, Twisk JW, et al., Hyposmia and executive dysfunction as predictors of future Parkinson's disease: a prospective study, Mov Disord 2009;24:1060-65.

17. Haehner A, Hummel T, Hummel C, et al., Olfactory loss may be a first sign of idiopathic Parkinson's disease, Mov Disord, 2007;22:839-42.

18. Sommer U, Hummel T, Cormann K, et al., Detection of presymptomatic Parkinson's disease: combination of olfactory tests, transcranial sonography, and 123 I-FP-CITSPECT, Mov Disord, 2004;19:1196-1202.

19. Marras $C$, Goldman S, Smith A, Smell identification ability in twin pairs discordant for Parkinson's disease, Mov Disord, 2005;20:687-93.

20. Ross W, Petrovitch $H$, Abbott RD ,et al., Association of Olfactory Dysfunction with Risk for Future Parkinson's Disease, Ann Neurol, 2008;63:167-73.

21. Hawkes $C$, The prodromal phase of sporadic Parkinson's disease: Does it exist and if so how long is it?, Mov Disord, 2008,23:47-53.

22. Braak H, Del Tredici K, Rub U, et al., Staging of brain pathology related to sporadic Parkinson's disease, Neurobiol Aging, 2003;24:197-211.

23. Huisman $E$, Uylings HB, Hoogland PV, A $100 \%$ increase of dopaminergic cells in the olfactory bulb may explain hyposmia in Parkinson's disease, Mov Disord, 2004;19: 687-92.

24. Huisman E, Uylings HB, Hoogland PV, Gender-Related Changes in Increase of Dopaminergic Neurons in the Olfactory Bulb of Parkinson's Disease Patients, Mov Disord 2008:23:1407-13.

25. Wenning GK, Shephard B, Hawkes C, et al., Olfactory function in atypical parkinsonian syndromes, Acta Neurol scand, 1995;91:247-50.

26. Hummel T, Sekinger B, Wolf SR, et al., "Sniffin' Sticks": Olfactory performance assessed by the combined testing of odor identification, odor discrimination and olfactory threshold, Chem Senses, 1997;22:39-52.

27. McKinnon JH, Demaerschalk BM, Caviness JN, et al. Sniffing out Parkinson disease: can olfactory testing differentiate parkinsonian disorders?, Neurologist, 2007:13:382-5.

28. Liberini P, Parola S, Spano PF, et al., Olfactory dysfunction in dementia associated with Lewy bodies, Parkinsonism Re Disord, 1999;5:30.
29. Williams SS, Williams J, Combrinck M, et al., Olfactory impairment is more marked in patients with mild dementia with Lewy bodies than those with mild Alzheimer disease, I Neurol Neurosurg Psychiatry, 2009;80:667-70

30. Hawkes $C$, Olfaction in neurodegenerative disorder, Mov Disord, 2003;18:364-72.

31. Katzenschlager R, Lees AJ, Olfaction and Parkinson's syndromes: its role in differential diagnosis, Curr Opin Neurol, 2004;17:417-23.

32. Silveira-Moriyama L, Guedes $L C$, Kingsbury $A$, et al., Hyposmia in G2019S LRRK2-related parkinsonism: clinical and pathologic data, Neurology, 2008;71:1021-6.

33. Doty RL, Singh A, Tetrud J, et al., Lack of major olfactory dysfunction in MPTP-induced parkinsonism, Ann Neurol, 1992;32:87-100

34. Miwa T, Watanabe A, Mitsumoto Y, et al., Olfactory impairment and Parkinson's disease-like symptoms observed in the common marmoset following administration of 1-methyl-4-phenyl-1,2,3,6tetrahydropyridine, Acta Otolaryngol Suppl, 2004;553:80-84.

35. Krüger S, Haehner A, Thiem C, et al., Neuroleptic-induced parkinsonism is associated with olfactory dysfunction, J Neurol, 2008;255:1574-9.

36. Müller A, Reuner U, Landis B, et al., Extrapyramidal symptoms in Wilson's disease are associated with olfactory dysfunction, Mov Disord, 2006;21:1311-16.

37. Witt M, Bormann K, Gudziol V, et al., Biopsies of olfactory epithelium in patients with Parkinson's disease, Mov Disord, 2009;24:906-14.

38. Müller A, Abolmaali ND, Hakimi AR, et al., Olfactory bulb volumes in patients with Idiopathic Parkinson's Disease a pilot study, I Neural Transm, 2005;112:1363-70.

39. Scherfler C, Schocke M, Seppi K, et al., Voxel-wise analysis of diffusion weighted imaging reveals disruption of the olfactory tract in Parkinson's disease, Brain, 2005;129:538-42.

40. Westermann B, Wattendorf E, Schwerdtfeger $U$, et al., Functional imaging of the cerebral olfactory system in patients with Parkinson's disease, J Neurol Neurosurg Psychiatry, 2008;79:19-24.

41. Wattendorf E, Welge-Lüssen A, Fiedler K, et al., Olfactory impairment predicts brain atrophy in Parkinson's disease, J Neuroscience, 2009;29:15410-13. 